OPEN ACCESS

Edited by:

Georg Jander,

Boyce Thompson Institute,

United States

Reviewed by:

Chi-Kuang Wen,

Shanghai Institutes for Biological

Sciences (CAS), China

Jie Le,

Institute of Botany (CAS), China

${ }^{*}$ Correspondence:

Joseph J. Kieber

jkieber@bio.unc.edu

Specialty section: This article was submitted to

Plant Physiology,

a section of the journal

Frontiers in Plant Science

Received: 20 September 2019 Accepted: 14 November 2019

Published: 05 December 2019

Citation:

Polko JK and Kieber JJ (2019) 1-Aminocyclopropane

1-Carboxylic Acid and Its Emerging Role as an EthyleneIndependent Growth Regulator.

Front. Plant Sci. 10:1602.

doi: 10.3389/fpls.2019.01602

\section{1-Aminocyclopropane 1-Carboxylic Acid and Its Emerging Role as an Ethylene-Independent Growth Regulator}

\author{
Joanna K. Polko and Joseph J. Kieber* \\ Department of Biology, University of North Carolina, Chapel Hill, NC, United States
}

1-Aminocyclopropane 1-carboxylic acid (ACC) is the direct precursor of the plant hormone ethylene. ACC is synthesized from S-adenosyl-L-methionine (SAM) by ACC synthases (ACSs) and subsequently oxidized to ethylene by ACC oxidases (ACOs). Exogenous ACC application has been used as a proxy for ethylene in numerous studies as it is readily converted by nearly all plant tissues to ethylene. However, in recent years, a growing body of evidence suggests that ACC plays a signaling role independent of the biosynthesis. In this review, we briefly summarize our current knowledge of ACC as an ethylene precursor, and present new findings with regards to the post-translational modifications of ACS proteins and to ACC transport. We also summarize the role of ACC in regulating plant development, and its involvement in cell wall signaling, guard mother cell division, and pathogen virulence.

Keywords: 1-aminocyclopropane 1-carboxylic acid, plant physiology, plant hormones, plant signaling, ethylene

\section{1-AMINOCYCLOPROPANE 1-CARBOXYLIC ACID AS A PRECURSOR OF ETHYLENE}

Four decades ago, 1-aminocyclopropane 1-carboxylic acid (ACC), a non-proteinogenic amino acid, was discovered to be an intermediate in the biosynthesis of the plant hormone ethylene (Adams and Yang, 1979). Ethylene regulates a wide range of developmental processes and responses to biotic and abiotic stresses, in part by complex interactions with other phytohormones (Muday et al., 2012; Vandenbussche et al., 2012; Merchante et al., 2013; Dubois et al., 2018). Its biosynthesis starts with the conversion of the amino acid methionine to $S$-adenosyl L-methionine (SAM) by SAM synthetase and the subsequent conversion of SAM to ACC, which is catalyzed by ACC synthase (ACS) (Figure 1) (Adams and Yang, 1977; Adams and Yang, 1979). The by-product of this reaction, 5'-methylthioadenosine (MTA), is recycled back into the Yang cycle while ACC is oxidized to ethylene by ACC oxidase (ACO) (Murr and Yang, 1975). In Arabidopsis, ACO proteins are encoded by five genes (ACO1-5), which belong to a superfamily of oxygenases/oxidases (Dong et al., 1992; Zhang et al., 2004). In general, ACS is the rate-limiting step in ethylene biosynthesis, though in some instances, ACO activity is limiting (Vriezen et al., 1999; Van de Poel et al., 2012). This topic, along with current knowledge on ACO phylogeny and their regulation and importance in agriculture, has been comprehensively discussed in a recent review (Houben and Van de Poel, 2019). 


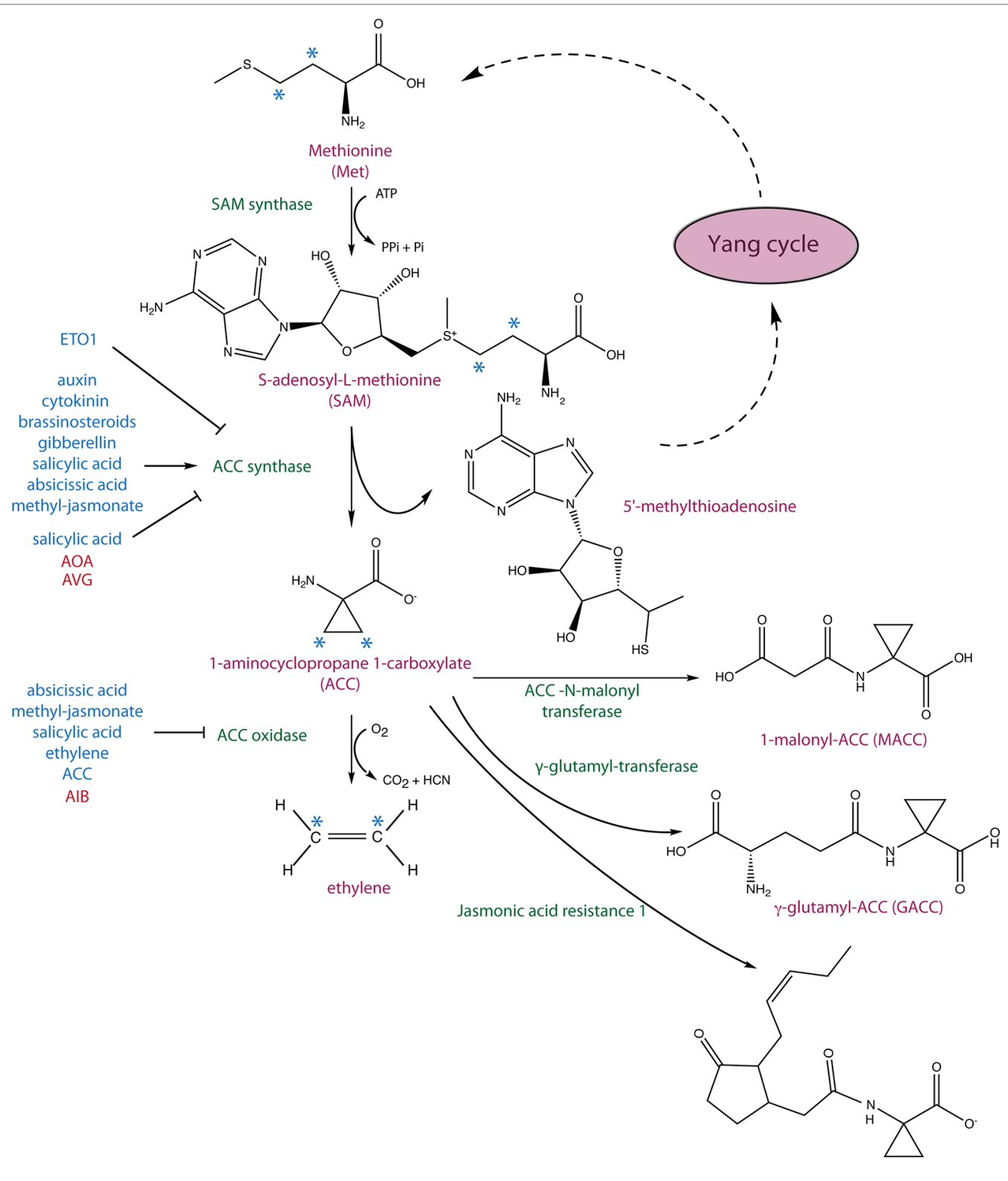

jasmonyl-ACC (JA-ACC)

FIGURE 1 | Ethylene biosynthetic pathway and 1-aminocyclopropane 1-carboxylic acid (ACC) conjugation. S-adenosyl-L-methionine (SAM) synthase converts methionine to SAM, which is subsequently converted to ACC and 5'-methylthioadenosine (MTA) by ACC synthase (ACS). MTA is recycled back to the Yang cycle to recover methionine, and ACC is oxidized to ethylene by ACC oxidase (ACO). The hormonal inputs that regulate ACS and ACO expression as well as ACS stability are depicted in blue. ACC has been shown to be converted to three derivates: 1-malonyl-ACC (MACC) by the ACC-N-malonyl transferase, $\gamma$-glutamyl-ACC by a glutamyl-transferase, and jasmonyl-ACC (JA-ACC) by jasmonic acid resistance1 (JAR1). The asterisks mark carbons that give rise to ethylene.

Conjugation of ACC has long been hypothesized to play a role in regulating the level of ethylene biosynthesis but may also generate novel signaling molecules. ACC can be conjugated to 1malonyl-ACC (MACC), $\gamma$-glutamyl-ACC (GACC), jasmonylACC (JA-ACC) (Amrhein et al., 1981; Martin et al., 1995; Staswick and Tiryaki, 2004) (Figure 1), and perhaps other yetto-be discovered conjugates. ACC conjugation regulates the availability of ACC to be converted to ethylene and, therefore, can be utilized by plants to control the level of ethylene biosynthesis. The ACC-to-MACC conversion is catalyzed by the enzyme ACC $N$-malonyl transferase (Martin et al., 1995; Peiser and Yang, 1998). MACC is the most abundant ACC conjugate in ripening Lycopersicon esculentum (tomato) fruit, and its formation can be induced by ethylene (Liu et al., 1985; Martin et al., 1995; Peiser and Yang, 1998). ACC was shown to be hydrolyzed back to ACC in Nasturtium officinale (watercress) stems, Nicotiana tabacum (tobacco) leaf discs, and senescing Dianthus caryophyllus (carnation) petals (Jiao et al., 1986; 
Hanley et al., 1989; ). The formation of GACC is catalyzed by the enzyme $\gamma$-glutamyl-transferase (GGT) (Martin et al., 1995; Martin and Slovin, 2000) which, in Arabidopsis, is encoded by four widely expressed GGT (1-4) genes, two of which (GGT3 and GGT4) encode catalytically inactive or minimally active enzymes. Interestingly, GGT1 and GGT2 appear to be localized extracellularly (Martin et al., 2007).

JA-ACC is the second most abundant JA conjugate detected in Arabidopsis leaves and is formed by JAR1, a JA-amino synthetase. Similar to MACC and GACC, JA-ACC might regulate levels of ACC available for the biosynthesis of ethylene, and may also regulate JA levels in the plant (Staswick and Tiryaki, 2004). The availability of ACC can also be controlled by plant and bacterial encoded ACC deaminases that irreversibly convert ACC to ammonia and $\alpha$-ketobutyric acid (Glick et al., 1998). Multiple species of plant growthpromoting bacteria from various phyla, including Proteobacteria, Actinobacteria, Firmicutes, and Bacteroidetes, contain ACC deaminases that can decrease host plant ACC levels. A decrease of ACC often facilitates stress-coping mechanisms under various unfavorable conditions (reviewed in Glick, 2014; Nascimento et al., 2014; Van de Poel and Van Der Straeten, 2014). For example, tomato plants grown in the presence of ACC deaminase-producing Enterobacter or Pseudomonas strains exhibit an increased tolerance to flooding stress, likely as a result of decreased ethylene (Grichko and Glick, 2001a). Moreover, Arabidopsis, Populus (aspen), and tomato plants have been shown to contain ACC deaminases, but their role in plant growth and development has not as yet been elucidated (McDonnell et al., 2009; Plett et al., 2009).

ACS is generally encoded by a multigene family in most plant species. For example, in Arabidopsis, ACS proteins are encoded by a family of 12 genes, though only ACS2-ACS9 and ACS11 encode functional ACS enzymes; ACS10 and ACS12 encode aminotransferases (Liang et al., 1995; Yamagami et al., 2003), ACS3 is a pseudogene, and ACS1 is catalytically inactive due to deletion of a highly conserved tripeptide Thr-Asn-Pro (TNP) (Liang et al., 1995). The remaining Arabidopsis ACS proteins can potentially form up to 45 different functional homo- and heterodimers, comprising a family of ACS enzymes with diverse biochemical properties (Tsuchisaka and Theologis, 2004; Tsuchisaka et al., 2009). ACS enzymes share an Nterminal catalytic domain and can be divided into three classes based on the presence of regulatory residues within their Ctermini (Harpaz-Saad et al., 2012). The C-terminal domain of type-1 ACSs (ACS2 and ACS6 in Arabidopsis) have target residues for both calcium-dependent and mitogen-activated protein kinases (CDPKs and MAPKs, respectively) (Liu and Zhang, 2004; Sebastià et al., 2004). Joo et al. (2008) showed that phosphorylation of the Ser residues within the C-terminus of ACS6 by MPK6 increases its stability and is associated with increased rates of ethylene production. Type- 2 ACS proteins have a target site for CDPKs and an overlapping Target of ETO1 (TOE) motif. Type-2 ACSs are targeted for degradation by the $26 \mathrm{~S}$ proteasome pathway upon binding of ETHYLENE
OVERPRODUCER1 (ETO1) or one of its paralogs, ETO-like1 or 2 (EOL1/2) (Chae et al., 2003; Wang et al., 2004; Christians et al., 2009; ). Phosphorylation of the C-terminus of type-2 ACS proteins reduces their targeting for degradation. Type-3 ACS proteins (ACS7 in Arabidopsis) have a short C-terminus that lacks an apparent regulatory domain, though ACS7 levels can be mediated through the activity of the E3 ligase XBAT32 (Prasad et al., 2010; Lyzenga et al., 2012). The 14-3-3 phospho-specific binding proteins bind various ACS isoforms in planta to regulate their stability. 14-3-3s also bind to ETO1 and EOL2, but in these cases, it results in their destabilization. Therefore, 14-3-3s control the level of ACS proteins through a bipartite mechanism-on one hand stabilizing them through direct binding, but also destabilizing the ubiquitin ligases involved in their degradation (Yoon and Kieber, 2013).

There is a complex crosstalk between ethylene and other plant hormones at the level of signaling and/or biosynthesis, the latter of which includes both transcriptional and posttranscriptional regulation of ACS (reviewed in Kazan and Manners, 2012; Muday et al., 2012; Van de Poel et al., 2015; Shigenaga and Argueso, 2016; Hu et al., 2017; Liu et al., 2017; Zemlyanskaya et al., 2018; Bürger and Chory, 2019; Qin et al., 2019). For example, cytokinin and brassinosteroid additively increase the stability of type-2 ACS proteins independently of their TOE domains (Hansen et al., 2009). The effect of various phytohormones on the rates of degradation of type-1, -2 , and -3 ACS proteins was comprehensively investigated using etiolated Arabidopsis seedlings as a model (Lee et al., 2017). Consistent with previous results, auxin was found to increase ACS2 and ACS5 transcript levels (type- 1 and type-2, respectively) as well as to stabilize their encoded proteins. Gibberellin, brassinosteroid, and cytokinin were also found to decrease the turnover of ACS2 and ACS5 proteins. Salicylic acid (SA) stabilized ACS5 but decreased the stability of ACS2 protein, the latter of which is different from the effect in light-grown seedlings in which SA stabilizes ACS2 (Liu and Zhang, 2004; Lee et al., 2017). Abscisic acid (ABA) and methyl-jasmonate (MeJA) did not affect ACS2 protein levels but increased the stability of ACS5, which is distinct from the negative effect of these hormones on levels of ethylene production (Lee et al., 2017). The reduced ethylene biosynthesis in response to ABA and MeJA is likely explained by the downregulation of $A C O$ genes in response to these hormones (Lee et al., 2017). Interestingly, the turnover of ACS7 (a type-3 ACS) was not regulated by any of the hormones examined in the study, and the analysis of its half-life showed that ACS7 is the most stable protein, confirming previous suggestions (Chae and Kieber, 2005). Moreover, the heterodimerization with ACS7 increased the stability of both ACS2 and ACS5 as compared to the respective homodimers, which suggests that dimerization among various ACS isoforms may regulate their turnover rate and, as a result, ethylene biosynthesis (Lee et al., 2017). ACS5 proteins are also stabilized when etiolated Arabidopsis seedlings are moved to the light, promoting ethylene biosynthesis and hypocotyl elongation during this transition (Seo and Yoon, 2019). 


\section{ACC TRANSPORT AND LYSINE HISTIDINE TRANSPORTERS}

Ethylene is involved in various stress-related responses such as wounding, pathogen infection, neighbor proximity, elevated temperatures, drought, soil waterlogging, and submergence (Vandenbussche et al., 2005; Sasidharan and Voesenek, 2015; Huang et al., 2016; Loreti et al., 2016; Valluru et al., 2016; Dubois et al., 2018). Following the demonstration that ethylene leads to epinasty of petioles in waterlogged tomato plants (Jackson and Campbell, 1975), Bradford and Yang showed that waterlogging and root anoxia correlated with the shootward transport of ACC, its subsequent conversion to ethylene, and leaf epinasty (Bradford and Yang, 1980). This spatial separation between the biosynthesis of ACC and the its conversion to ethylene is the result of the oxygen dependence of the ACO enzyme (Murr and Yang, 1975). Multiple studies confirmed the phenomenon of ACC transport between roots and shoots in several plant species (e.g. Else and Jackson, 1998). During the de-submergence of flood-tolerant Rumex palustris (marsh dock) plants, ACC delivered from the root contributes to the pool of ACC that accumulated in the shoot during submergence to stimulate petiole elongation (Voesenek et al., 2003). In contrast, floodintolerant Rumex acetosa (common sorrel) does not accumulate ACC in roots or shoots and consequently fails to recover from the detrimental effects of flooding. The root-to-shoot transport of ACC is thought to occur primarily in the xylem, though there is evidence for phloem-translocated ACC as well (Amrhein et al., 1981; Hume and Lovell, 1983). Radio-labeled ACC application to the abaxial side of Gossypium hirsutum (cotton) leaves resulted in both basipetal and acropetal transport of ACC throughout the plant as well as rapid conversion to $\left[{ }^{14} \mathrm{C}\right] \mathrm{MACC}$, which was not translocated from the source leaf. ACC is compartmentalized within the tonoplast of Zea mays (maize) leaf mesophyll cells via a mechanism dependent on an electrochemical gradient (Saftner and Martin, 1993). Translocation of ACC conjugates into the vacuole likely plays a role in regulating ACC availability and/or ethylene levels. In Acer pseudoplatanus (sycamore maple) protoplasts treated with $\left[{ }^{14} \mathrm{C}\right] \mathrm{ACC}$, there was a steady transport of the $\left[{ }^{14} \mathrm{C}\right] \mathrm{MACC}$ conjugate into the vacuole (Bouzayen et al., 1988). Furthermore, Tophof et al. (1989) showed that MACC accumulated to higher levels than ACC in vacuoles in both wheat (Triticum aestivum) and barley (Hordeum vulgare) plants.

The ability of plants to transport ACC both within the cell and throughout the plant suggests the existence of ACC transporters. ACC and its structural analog $\alpha$-aminoisobutyric acid (AIB) are taken up by tomato pericarp cells; this uptake is inhibited by neutral but not by acidic or basic amino acids (Saftner and Baker, 1987). Tophof et al. (1989) speculated that ACC might be translocated to the tonoplast by a neutral amino acid transporter and as MACC competitively inhibited the transport of malate to the vacuole, they suggested that these molecules likely shared a common translocator. Recently, the identification of an ACC-resistant (are2) Arabidopsis mutant that displayed a reduced uptake of exogenous ACC led to the identification of the LYSINE HISTIDINE TRANSPORTER1 (LHT1) as a potential ACC transporter (Shin et al., 2014). LHT1 localized to the plasma membrane of leaf mesophyll and epidermal root cell and is not detected in the vasculature (Chen and Bush, 1997; Hirner et al., 2006). The lht1 mutant displays severe growth defects on media with aspartate and glutamate as the sole nitrogen source and is impaired in the uptake of $\left[{ }^{14} \mathrm{C}\right]$ labeled amino acids. The are 2 mutant, allelic to $h t 1-5$, was resistant to ACC but displayed a normal triple response when exposed to ethylene. Isolated are2/lht1-5 protoplasts display reduced accumulation of $\left[{ }^{14} \mathrm{C}\right] \mathrm{ACC}$. Additionally, competition experiments showed that the presence of alanine and glycine can reduce the triple response morphology elicited in response to ACC, consistent with Tophof's (1989) speculation that ACC is translocated through the tonoplast by a neutral amino acid transporter. However, Hirner et al. (2006) showed that lysine and histidine are the best substrates for LHT1, suggesting that multiple distinct transporters may act in the movement of ACC. It is possible that the ACC uptake and transport are mechanistically different and require distinct transport proteins. The subject of alternative ACC transporters and strategies to identify them has been recently discussed in a comprehensive review (Vanderstraeten and Van Der Straeten, 2017). Further studies are needed to identify and distinguish transporters involved in the short- and long-distance ACC translocation, its uptake into cells, as well as its intracellular trafficking.

\section{ACC IN PLANT DEVELOPMENT AND BEYOND}

A growing body of evidence indicates a role for ACC as a signaling molecule distinct from its role in ethylene biosynthesis. One of the first findings consistent with this was the discovery of the involvement of ACC in the regulation of cell wall function in the FEI pathway (Xu et al., 2008). FEI1 and FEI2 are leucine-rich repeat receptor-like kinases (LRR-RLKs) that have been linked to cellulose biosynthesis. fei1 fei2 loss-offunction mutants display root swelling under high concentrations of salt and sucrose, decreased biosynthesis of cellulose, hypersensitivity to the cellulose inhibitor isoxaben, thickening of etiolated hypocotyls, and a decrease in the formation of cellulose rays in seed coat mucilage ( $\mathrm{Xu}$ et al., 2008; Harpaz-Saad et al., 2011), which together indicate a role of the FEI proteins in regulating cellulose biosynthesis. Intriguingly, inhibition of ethylene biosynthesis [via aminooxy-acetic acid (AOA) or AIB; Figure 1] reverted the swollen root phenotype of feil fei2 mutants, but blocking ethylene perception, using either the inhibitors 1-methylcyclopropane (1-MCP) or silver thiosulfate, or by introducing ethylene-insensitive ein2 and etr 1 mutations into the feil fei2 background, had no effect. Furthermore, the FEI kinase domain was shown to directly interact with type-2 ACS proteins, suggesting a direct link to ACC synthesis (Xu et al., 2008). 
An analysis of cell elongation in roots treated with the cellulose biosynthesis inhibitor isoxaben provided further support that ACC acts as a signal. Tsang et al. (2011) analyzed root trichoblast length upon treatment with isoxaben in presence or absence of various ethylene inhibitors. Interestingly, inhibitors of ethylene biosynthesis [amino-ethoxyvinylglycine (AVG), AOA, or 2-anilino-7-(4-methoxy-phenyl)-7,8-dihydro-5(6H)quinazolinone] reversed the isoxaben-induced elongation defects, but inhibitors of ethylene perception did not. Moreover, short-term ACC treatment induced shortening of trichoblasts in ethylene-insensitive ein 3 eill mutants, further supporting the hypothesis that ACC acts independently of ethylene signaling. Additionally, the authors found that both cell wall damage-induced and ACC-mediated growth inhibition is dependent on auxin signaling since the growth inhibition was absent when combined with $\alpha$-(phenylethyl-2-one)- indole-3acetic acid (PEO-IAA), a transport inhibitor response 1 (TIR1) receptor antagonist This is consistent with the suppression of the fei1 fei2 root swelling phenotype by auxin biosynthesis mutants (Steinwand et al, 2014). Together, these studies suggest that ACC plays a role in the response to cell wall perturbations, triggered by either chemical or genetic disruption of cellulose synthesis, and that auxin is involved in this pathway (Xu et al., 2008; Tsang et al., 2011).
Genetic analysis of disruption of ACS genes in Arabidopsis also supports a function for ACC in addition to its role as an ethylene biosynthetic precursor. A comprehensive genetic study of all members of the ACS gene family in Arabidopsis, including the generation and analysis of single, double, triple, and highorder acs mutants, suggested novel roles for ACS beyond ethylene biosynthesis (Tsuchisaka et al., 2009). Analysis of the mutants revealed both synergistic and antagonistic relationships among various ACS genes in ethylene biosynthesis and in regulation of hypocotyl and rosette growth, and flowering time. Disruption of multiple $A C S$ genes led to a progressive increase in plant size, concomitant with a decreased level of ethylene biosynthesis. Remarkably, an octuple acs 2, 4, 5, 6, 7, 9, amiR acs 8 acs 11 mutant, which had a $\sim 90 \%$ decrease in the level of ethylene production, displayed embryonic/gametophytic lethality and/or unfertilized ovules. The octuple acs mutant inflorescences are significantly taller than wild-type or lowerorder acs mutants, despite their initial reduced growth rate. An independent octuple mutant line analyzed in the study could only be propagated when the amiR transgene was heterozygous, consistent with embryo/gametophytic lethality or infertility. The striking phenotypes of the octuple acs mutant is distinct from the full reproductive viability of even very strong ethylene signaling mutants, suggesting that $A C S$ genes play a role beyond acting as

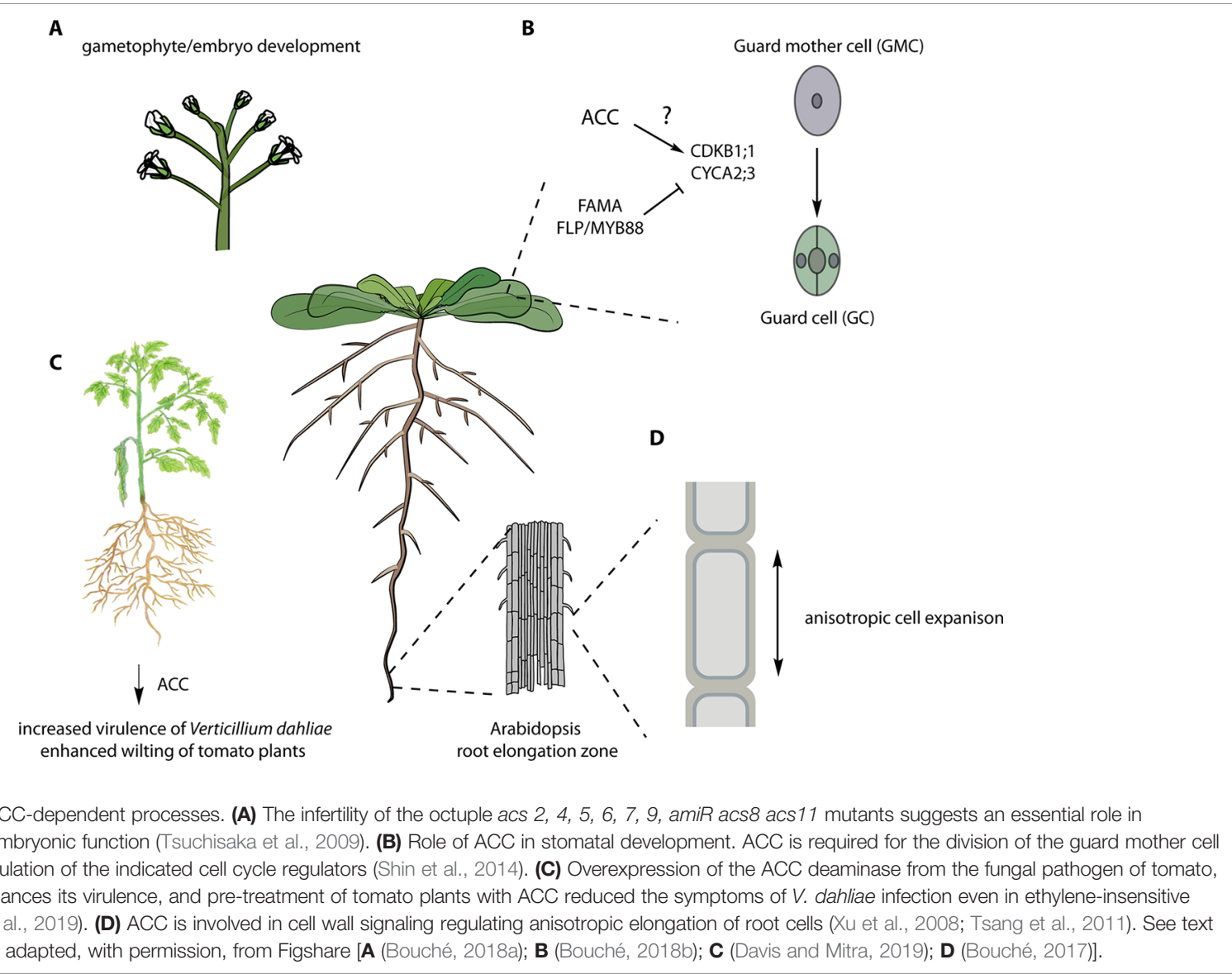


precursors in ethylene biosynthesis. Alternatively, ACS proteins may have a moonlighting function, and the reported lethality of the octuple mutants may result from disruption of an unrelated process. The precise nature of the embryo/gametophyte lethality of the octuple acs mutant needs further characterization.

ACC was recently shown to play a role in stomatal development. The terminal division of the guard mother cell (GMC) produces the two guard cells (GCs) that comprise the mature stomata (Yin et al., 2018). Application of the ACS inhibitor AVG induced the formation of pore-less, single guard cell (SGCs). Similarly, the octuple acs 2, 4, 5, 6, 7, 9, amiR acs8 acs11 mutant (Tsuchisaka et al., 2009) developed SGCs. This SGC phenotype was not observed in the presence of ACO inhibitors (AIB or $\mathrm{Co}^{2+}$ ) or the ethylene binding inhibitor 1$\mathrm{MCP}$, nor was it present in various ethylene-signaling mutants (etr1, ein2, ctr1, ein3 eil2). ACC did not increase cell division in GMCs in wild-type plants but did so (as did ethylene) in fama and four lips $(f l p) / m y b 88$ mutants, which developed clusters of thin cells on the epidermis. FAMA and FOUR LIPS (FLP)/ MYB88 are central regulators of the last cell division of GMC, acting upstream of the core cell cycle genes (CYCA2;3, CDKB1;1, and $C D K A ; 1)$ (Xie et al., 2010; Vanneste et al., 2011; Yang et al., 2014). Because ACC induced extra divisions in these mutant backgrounds, it was concluded that FAMA and FLP/MYB88 might antagonize the effect of ACC on the GMC division. Moreover, ACC, but not ethylene, stimulated the expression of CYCA2;3 and CDKB1;1 in fama and flp/my88 mutants, and conversely, AVG downregulated the expression of these genes. ACC partially rescued the SGC formation in the acs 2, 4, 5, 6, 7, 9, amiR acs 8 acs11 line, but not in cyca2;3 and cdkb1;1 mutants, suggesting that it acts upstream of the cell cycle-dependent control of the GMC division.

Recent studies suggest that the signaling role of ACC could extend beyond the plant kingdom. Since many plant growthpromoting rhizobacteria (PGPR) possess ACC deaminase genes and utilize ACC as a source of nitrogen, Li et al. (2019) examined whether ACC could act as a chemoattractant. Indeed, Pseudomonas putida displayed a chemotactic response to ACC, but not to ethylene, and the ability to respond to ACC was correlated to the ability of $P$. putida to colonize wheat roots.

The fungal pathogen, Verticillium dahliae is a soil-borne pathogen of many plant species, causing vascular wilt disease. Tsolakidou et al. (2019) found that genetic modulation of ACC levels in $V$. dahliae affected its microsclerotia development and hyphae growth. Overexpression of ACC deaminase in V. dahliae led to increased virulence on tomato and eggplant, including enhanced wilting and greater fungal growth. On the contrary, the

\section{REFERENCES}

Adams, D. O., and Yang, S. F. (1977). Methionine metabolism in apple tissue implication of S-adenosylmethionine as an intermediate in conversion of methionine to ethylene. Plant Physiol. 60, 892-896. doi: 10.1104/pp.60.6.892

Adams, D. O., and Yang, S. F. (1979). Ethylene biosynthesis - identification of 1aminocyclopropane-1-carboxylic acid as an intermediate in the conversion of methionine to ethylene. Proc. Natl. Acad. Sci. U.S.A. 76, 170-174. doi: 10.1073/ pnas.76.1.170 disruption of ACC deaminase in $V$. dahliae resulted in reduced virulence and less fungal biomass. To test if ACC was acting as a signal controlling plant defense, wild-type and the ethyleneinsensitive mutant Never-ripe $(\mathrm{Nr})$ tomato plants were treated with ACC and then infected with $V$. dahliae. ACC increased the resistance of both wild-type and $N r$ plants, suggesting that ACC and not ethylene promotes disease resistance against $V$. dahliae. It will be interesting to investigate if similar responses occur in other plant-pathogen interactions.

\section{CONCLUSIONS AND OUTSTANDING QUESTIONS}

An increasing number of studies have established that ACC acts as a signaling molecule beyond its function in ethylene biosynthesis (Figure 2). ACC appears to be involved in regulating multiple processes, including stress responses, cell expansion, cell wall function, stomatal development, pathogen interactions, and fertilization-related events. Additional studies are needed to elucidate the mode of action of ACC and to further define its role in plant growth and development. For example, the embryo/gametophyte lethality of the octuple acs mutants (Tsuchisaka et al., 2009) raises questions about the precise basis for ACC action during gametophyte development, fertilization, or embryogenesis. The recent link between LHT and ACC uptake (Shin et al., 2014) provides tantalizing clues to the mechanisms of ACC uptake/transport, but as LHT is part of a large gene family, other amino acid transporters may also participate in ACC uptake and translocation (reviewed in Vanderstraeten and Van Der Straeten, 2017). The biological roles of ACC derivatives, including the JA-ACC, GACC, and MACC, also need to be investigated. Finally, in light of the findings presented in this review, studies that use ACC as a proxy for ethylene need to be interpreted with caution.

\section{AUTHOR CONTRIBUTIONS}

Both authors wrote the manuscript.

\section{FUNDING}

This work has been supported by grant IOS-1856431 from the National Science Foundation.

Amrhein, N., Schneebeck, D., Skorupka, H., Tophof, S., and Stockigt, J. (1981). Identification of a major metabolite of the ethylene precursor 1aminocyclopropane-1-carboxylic acid in higher-plants. Naturwissenschaften 68, 619-620. doi: 10.1007/BF00398617

Bürger, M., and Chory, J. (2019). Stressed out about hormones: how plants orchestrate immunity. Cell Host Microbe. 26, 163-172. doi: 10.1016/ j.chom.2019.07.006

Bouché, F. (2017). Arabidopsis - Root cell types. figshare. Figure. doi: 10.6084/ m9.figshare.4688752.v1 
Bouché, F. (2018a). 2018_Arabidopsis_inflorescence_zoom. figshare. Figure. doi: 10.6084/m9.figshare.7159949.v1

Bouché, F. (2018b). 2018_Arabidopsis_flowering_plant. figshare. Figure. doi: 10.6084/m9.figshare.7159937.v1

Bouzayen, M., Latche, A., Alibert, G., and Pech, J. C. (1988). Intracellular sites of synthesis and storage of 1-(malonylamino) cyclopropane-1-carboxylic acid in acer-pseudoplatanus cells. Plant Physiol. 88, 613-617. doi: 10.1104/pp.88.3.613

Bradford, K. J., and Yang, S. F. (1980). Xylem transport of 1-aminocyclopropane1-carboxylic acid, an ethylene precursor, in waterlogged tomato plants. Plant. Physiol. 65, 322-326. doi: 10.1104/pp.65.2.322

Chae, H. S., and Kieber, J. J. (2005). Eto Brute? Role of ACS turnover in regulating ethylene biosynthesis. Trends Plant Sci. 10, 291-296. doi: 10.1016/ j.tplants.2005.04.006

Chae, H. S., Faure, F., and Kieber, J. J. (2003). The eto1, eto2, and eto3 mutations and cytokinin treatment increase ethylene biosynthesis in Arabidopsis by increasing the stability of ACS protein. Plant Cell 15, 545-559. doi: 10.1105/ tpc. 006882

Chen, L., and Bush, D. R. (1997). LHT1, a lysine- and histidine-specific amino acid transporter in Arabidopsis. Plant Physiol. 115, 1127-1134. doi: 10.1104/ pp.115.3.1127

Christians, M. J., Gingerich, D. J., Hansen, M., Binder, B. M., Kieber, J. J., and Vierstra, R. D. (2009). The BTB ubiquitin ligases ETO1, EOL1 and EOL2 act collectively to regulate ethylene biosynthesis in Arabidopsis by controlling type-2 ACC synthase levels. Plant J. 57, 332-345. doi: 10.1111/j.1365313X.2008.03693.x

Davis, D., and Mitra, M. (2019). Wilting tomato plants. figshare. Figure. doi: 10.6084/m9.figshare.8049965.v1

Dong, J. G., Fernandezmaculet, J. C., and Yang, S. F. (1992). Purification and characterization of 1-aminocyclopropane-1-carboxylate oxidase from apple fruit. Proc. Natl. Acad. Sci. U.S.A. 89, 9789-9793. doi: 10.1073/pnas.89.209789

Dubois, M., Van den Broeck, L., and Inzé, D. (2018). The pivotal role of ethylene in plant growth. Trends Plant Sci. 23, 1-14. doi: 10.1016/j.tplants.2018.01.003

Else, M. A., and Jackson, M. B. (1998). Transport of 1-aminocyclopropane-1carboxylic acid (ACC) in the transpiration stream of tomato (Lycopersicon esculentum) in relation to foliar ethylene production and petiole epinasty. Functional Plant Biol. 25, 453-457. doi: 10.1071/PP97105

Glick, B., Penrose, D., and Li, J. (1998). A model for the lowering of plant ethylene concentrations by plant growth-promoting bacteria. J. Theor. Biol. 190, 63-68. doi: 10.1006/jtbi.1997.0532

Glick, B. R. (2014). Bacteria with ACC deaminase can promote plant growth and help to feed the world. Microbiol. Res. 169, 30-39. doi: 10.1016/ j.micres.2013.09.009

Grichko, V. P., and Glick, B. R. (2001a). Amelioration of flooding stress by ACC deaminase-containing plant growth-promoting bacteria. Plant Physiol. Biochem. 39, 11-17. doi: 10.1016/S0981-9428(00)01212-2

Hanley, K. M., Meir, S., and Bramlage, W. J. (1989). Activity of ageing carnation flower parts and the effects of 1-(malonylamino) cyclopropane-1-carboxylic acid-induced ethylene. Plant Physiol. 91, 1126-1130. doi: 10.1104/pp.91.3.1126

Hansen, M., Chae, H. S., and Kieber, J. J. (2009). Regulation of ACS protein stability by cytokinin and brassinosteroid. Plant J. 57, 606-614. doi: 10.1111/ j.1365-313X.2008.03711.x

Harpaz-Saad, S., McFarlane, H. E., Xu, S., Divi, U. K., Forward, B., Western, T. L., et al. (2011). Cellulose synthesis via the FEI2 RLK/SOS5 pathway and CELLULOSE SYNTHASE 5 is required for the structure of seed coat mucilage in Arabidopsis. Plant J. 68, 941-953. doi: 10.1021/bm060292h

Harpaz-Saad, S., Yoon, G. M., Mattoo, A. K., and Kieber, J. J. (2012). The formation of ACC and competition between polyamines and ethylene for SAM. Annu. Plant Rev. 44, 53-81. doi: 10.1002/9781118223086.ch3

Hirner, A., Ladwig, F., Stransky, H., Okumoto, S., Keinath, M., Harms, A., et al. (2006). Arabidopsis LHT1 is a high-affinity transporter for cellular amino acid uptake in both root epidermis and leaf mesophyll. Plant Cell 18, 1931-1946. doi: 10.1105/tpc.106.041012

Houben, M., and Van de Poel, B. (2019). 1-Aminocyclopropane-1-Carboxylic Acid Oxidase (ACO): the enzyme that makes the plant hormone ethylene. Front. Plant Sci. 10, 695. doi: 10.3389/fpls.2019.00695

Hu, Y., Vandenbussche, F., and Van Der Straeten, D. (2017). Regulation of seedling growth by ethylene and the ethylene-auxin crosstalk. Planta 245, 467489. doi: 10.1007/s00425-017-2651-6
Huang, P.-Y., Catinot, J., and Zimmerli, L. (2016). Ethylene response factors in Arabidopsis immunity. J. Exp. Bot. 67, 1231-1241. doi: 10.1093/jxb/erv518

Hume, B., and Lovell, P. (1983). Role of aminocyclopropane-1-carboxylic acid in ethylene release by distal tissues following localized application of ethephon in. Cucurbita pepo. Physiol. Plant. 58, 101- 106 (1983. doi: 10.1080/ 03015521.1981.10427831

Jackson, M. B., and Campbell, D. J. (1975). Movement of ethylene from roots to shoots, a factor in the responses of tomato plants to waterlogged soil conditions. New Phytol. 74, 397- 406. doi: 10.1111/j.1469-8137.1975. tb01350.x

Jiao, X. Z., Philosoph-Hadas, S., Su, L. Y., and Yang, S. F. (1986). The conversion of 1-(malonylamino) cyclopropane-1-carboxylic acid to 1-aminocyclopropane-1carboxylic acid in plant tissues. Plant Physiol. 81, 637-641. doi: 10.1104/ pp.81.2.637

Joo, S., Liu, Y., Lueth, A., and Zhang, S. (2008). MAPK phosphorylation-induced stabilization of ACS6 protein is mediated by the non-catalytic C-terminal domain, which also contains the cis-determinant for rapid degradation by the 26 S proteasome pathway. Plant J. 54, 129-140. doi: 10.1111/j.1365313X.2008.03404.x

Kazan, K., and Manners, J. M. (2012). JAZ repressors and the orchestration of phytohormone crosstalk. Trends Plant Sci. 17, 22-31. doi: 10.1016/ j.tplants.2011.10.006

Lee, H. Y., Chen, Y.-C., Kieber, J. J., and Yoon, G. M. (2017). Regulation of the turnover of ACC synthases by phytohormones and heterodimerization in Arabidopsis. Plant J. 91, 491-504. doi: 10.1111/tpj.13585

Li, T., Zhang, J., Shen, C., Li, H., and Qiu, L. (2019). 1-Aminocyclopropane-1carboxylate: a novel and strong chemoattractant for the plant beneficial rhizobacterium Pseudomonas putida UW4. Mol. Plant Microbe Interact. 32, 750-759. doi: 10.1094/MPMI-11-18-0317-R

Liang, X., Oono, Y., Shen, N. F., Köhler, C., Li, K., Scolnik, P. A., et al. (1995). Characterization of two members (ACS1 and ACS3) of the 1aminocyclopropane-1-carboxylate synthase gene family of Arabidopsis thaliana. Gene 167, 17-24. doi: 10.1016/0378-1119(95)00694-x

Liu, Y., and Zhang, S. (2004). Phosphorylation of 1-aminocyclopropane-1carboxylic acid synthase by mpk6, a stress-responsive mitogen-activated protein kinase, induces ethylene biosynthesis in Arabidopsis. Plant Cell 16, 3386-3399. doi: 10.1105/tpc.104.026609

Liu, Y., Su, L. Y., and Yang, S. F. (1985). Ethylene promotes the capability to malonylate 1-aminocyclopropane-1-carboxylic acid and d-amino acids in preclimacteric tomato fruits. Plant Phyiol. 77, 891-895. doi: 10.1104/ pp.77.4.891

Liu, J., Moore, S., Chen, C., and Lindsey, K. (2017). Crosstalk complexities between auxin, cytokinin, and ethylene in Arabidopsis root development: from experiments to systems modeling, and back again. Mol. Plant 10, 1480-1496. doi: 10.1016/j.molp.2017.11.002

Loreti, E., van Veen, H., and Perata, P. (2016). Plant responses to flooding stress. Curr. Opin. Plant Biol. 33, 64-71. doi: 10.1016/j.pbi.2016.06.005

Lyzenga, W. J., Booth, J. K., and Stone, S. L. (2012). The Arabidopsis RING-type E3 ligase XBAT32 mediates the proteasomal degradation of the ethylene biosynthetic enzyme, 1-aminocyclopropane-1-carboxylate synthase 7. Plant J. 71, 23-34. doi: 10.1111/j.1365-313X.2012.04965.x

Martin, M. N., and Slovin, J. P. (2000). Purified $\gamma$-glutamyl transpeptidases from tomato exhibit high affinity for glutathione and glutathione S-conjugates. Plant Physiol. 122, 1417-1426. doi: 10.1104/pp.122.41417

Martin, M. N., Cohen, J. D., and Saftner, R. A. (1995). A new 1aminocyclopropane- 1-carboxylic acid-conjugating activity in tomato fruit. Plant Physiol. 109, 917-926. doi: 10.1104/pp.109.3.917

Martin, M. N., Saladores, P. H., Lambert, E., Hudson, A. O., and Leustek, T. (2007). Localization of members of the $\gamma$-glutamyl transpeptidase family identifies sites of glutathione and glutathione s-conjugate hydrolysis. Plant Physiol. 144, 1715-1732. doi: 10.1104/pp.106.094409

McDonnell, L., Plett, J. M., Andersson-Gunnerås, S., Kozela, C., Dugardeyn, J., Van Der Straeten, D., et al. (2009). Ethylene levels are regulated by a plant encoded 1-aminocyclopropane-1-carboxylic acid deaminase. Physiol. Plantarum 136, 94-109. doi: 10.1111/j.1399-3054.2009.01208.x

Merchante, C., Alonso, J. M., and Stepanova, A. N. (2013). Ethylene signaling: simple ligand, complex regulation. Curr. Opin. Plant Biol. 16, 554-560. doi: 10.1016/j.pbi.2013.08.001 
Muday, G. K., Rahman, A., and Binder, B. M. (2012). Auxin and ethylene: collaborators or competitors? Trends Plant Sci. 17, 181-195. doi: 10.1016/ j.tplants.2012.02.001

Murr, D. P., and Yang, S. F. (1975). Conversion of 5'-methylthioadenosine to methionine by apple tissue. Phytochemistry 14, 1291-1292. doi: 10.1016/ S0031- 9422(00)98613- 8

Nascimento, F. X., Rossi, M. J., Soares, C. R. F. S., McConkey, B. J., and Glick, B. R. (2014). New insights into 1-aminocyclopropane-1-carboxylate (ACC) deaminase phylogeny, evolution and ecological significance. PLoS One 9, e99168. doi: 10.1371/journal.pone.0099168

Peiser, G., and Yang, S. F. (1998). Evidence for 1-(malonylamino) cyclopropane1 - carboxylic acid being the major conjugate of aminocyclopropane-1carboxylic acid in tomato fruit. Plant Physiol. 116, 1527-1532. doi: 10.1104/ pp.116.41527

Plett, J. M., McDonnell, L., and Regan, S. (2009). Plant encoded 1-aminocyclopropane1 -carboxylic acid deaminase activity implicated in different aspects of plant development. Plant Signal. Behav. 4, 1186-1189. doi: 10.4161/psb.4.12.10060

Prasad, M. E., Schofield, A., Lyzenga, W., Liu, H., and Stone, S. L. (2010). Arabidopsis RING E3 ligase XBAT32 regulates lateral root production through its role in ethylene biosynthesis. Plant Physiol. 153, 1587-1596. doi: 10.1104/pp.110.156976

Qin, H., He, L., and Huang, R. (2019). The coordination of ethylene and other hormones in primary root development. Front. Plant Sci. 10, 444-448. doi: 10.3389/fpls.2019.00874

Saftner, R. A., and Baker, J. E. (1987). Transport and compartmentation of 1aminocyclopropane-1-carboxylic acid and its structural analog, alphaaminoisobutyric acid, in tomato pericarp slices. Plant Physiol. 84, 311-317. doi: $10.1104 / p p .84 .2 .311$

Saftner, R. A., and Martin, M. N. (1993). Transport of 1-aminocyclopropane- 1carboxylic acid into isolated maize mesophyll vacuoles. Physiol. Plant. 87, 535543. doi: 10.1111/j.1399-3054.1993.tb02504.x

Sasidharan, R., and Voesenek, L. A. C. J. (2015). Ethylene-mediated acclimations to flooding stress. Plant Physiol. 169, 3-12. doi: 10.1104/pp.15.00387

Sebastià, C. H., Hardin, S. C., Clouse, S. D., Kieber, J. J., and Huber, S. C. (2004). Identification of a new motif for CDPK phosphorylation in vitro that suggests ACC synthase may be a CDPK substrate. Arch. Biochem. Biophys. 428, 81-91. doi: 10.1016/j.abb.2004.04.025

Seo, D. H., and Yoon, G. M. (2019). Light-induced stabilization of ACS contributes to hypocotyl elongation during the dark-to-light transition in Arabidopsis seedlings. Plant J. 98, 898-911. doi: 10.1111/tpj.14289

Shigenaga, A. M., and Argueso, C. T. (2016). No hormone to rule them all: Interactions of plant hormones during the responses of plants to pathogens. Semin. Cell Dev. Biol. 56, 174-189. doi: 10.1016/j.semcdb.2016.06.005

Shin, K., Lee, S., Song, W.-Y., Lee, R.-A., Lee, I., Ha, K., et al. (2014). Genetic identification of ACC-RESISTANT2 reveals involvement of LYSINE HISTIDINE TRANSPORTER1 in the uptake of 1-aminocyclopropane-1-carboxylic acid in Arabidopsis thaliana. Plant Cell Physiol. 56, 572-582. doi: 10.1093/pcp/pcu201

Staswick, P. E., and Tiryaki, I. (2004). The oxylipin signal jasmonic acid is activated by an enzyme that conjugates it to isoleucine in Arabidopsis. Plant Cell 16, 2117-2127. doi: 10.1105/tpc.104.023549

Tophof, S., Martinoia, E., Kaiser, G., Hartung, W., and Amrhein, N. (1989). Compartmentation and transport of 1-aminocyclopropane-1-carboxylic acid and n-malonyl-1-aminocyclopropane-1-carboxylic acid in barley and wheat mesophyll-cells and protoplasts. Physiol. Plant. 75, 333-339. doi: 10.1111/j. 1399- 3054.1989.tb04635.x

Tsang, D. L., Edmond, C., Harrington, J. L., and Nühse, T. S. (2011). Cell wall integrity controls root elongation via a general 1-aminocyclopropane-1carboxylic acid-dependent, ethylene-independent pathway. Plant Physiol. 156, 596-604. doi: 10.1104/pp.111.175372

Tsolakidou, M.-D., Pantelides, L. S., Tzima, A. K., Kang, S., Paplomatas, E. J., and Tsaltas, D. (2019). Disruption and overexpression of the gene encoding acc (1aminocyclopropane-1-carboxylic acid) deaminase in soil-borne fungal pathogen Verticillium dahlia revealed the role of ACC as a potential regulator of virulence and plant defense. Mol. Plant Microbe Interact 32, 639-653. doi: 10.1094/MPMI-07-18-0203-R

Tsuchisaka, A., and Theologis, A. (2004). Heterodimeric interactions among the 1amino-cyclopropane-1-carboxylate synthase polypeptides encoded by the
Arabidopsis gene family. Proc. Natl. Acad. Sci. U.S.A. 101, 2275-2280. doi: 10.1073/pnas.0308515101

Tsuchisaka, A., Yu, G., Jin, H., Alonso, J. M., Ecker, J. R., Zhang, X., et al. (2009). A combinatorial interplay among the 1-aminocyclopropane-1-carboxylate isoforms regulates ethylene biosynthesis in Arabidopsis thaliana. Genetics 183, 979-1003. doi: 10.1534/genetics.109.107102

Valluru, R., Reynolds, M. P., Davies, W. J., and Sukumaran, S. (2016). Phenotypic and genome-wide association analysis of spike ethylene in diverse wheat genotypes under heat stress. New Phytol. 214, 271-283. doi: 10.1111/nph.14367

Van de Poel, B., and Van Der Straeten, D. (2014). 1-aminocyclopropane-1carboxylic acid (ACC) in plants: more than just the precursor of ethylene!. Front. Plant Sci. 5, 640. doi: 10.3389/fpls.2014.00640

Van de Poel, B., Bulens, I., Markoula, A., Hertog, M. L. A. T. M., Dreesen, R., Wirtz, M., et al. (2012). Targeted systems biology profiling of tomato fruit reveals coordination of the Yang cycle and a distinct regulation of ethylene biosynthesis during postclimacteric ripening. Plant Physiol. 160, 1498-1514. doi: $10.1104 /$ pp.112.206086

Van de Poel, B., Smet, D., and Van Der Straeten, D. (2015). Ethylene and hormonal cross talk in vegetative growth and development. Plant Physiol 169, 61-72. doi: 10.1104/pp.15.00724

Vandenbussche, F., Pierik, R., Millenaar, F. F., Voesenek, L. A., and Van Der Straeten, D. (2005). Reaching out of the shade. Curr. Opin. Plant Biol. 8, 462468. doi: 10.1016/j.pbi.2005.07.007

Vandenbussche, F., Vaseva, I., Vissenberg, K., and Van Der Straeten, D. (2012). Ethylene in vegetative development: a tale with a riddle. New Phytol. 194, 895909. doi: 10.1111/j.1469-8137.2012.04100.x

Vanderstraeten, L., and Van Der Straeten, D. (2017). Accumulation and transport of 1-aminocyclopropane-1-carboxylic acid (acc) in plants: current status, considerations for future research and agronomic applications. Front. Plant Sci. 8, 157-118. doi: 10.3389/fpls.2017.00038

Vanneste, S., Coppens, F., Lee, E., Donner, T. J., Xie, Z., Van Isterdael, G., et al. (2011). Developmental regulation of CYCA2s contributes to tissue-specific proliferation in Arabidopsis. EMBO J. 30, 3430-3441. doi: 10.1038/emboj. 2011.240

Voesenek, L. A. C. J., Jackson, M. B., Toebes, A. H. W., Huibers, W., Vriezen, W. H., and COLMER, T. D. (2003). De-submergence-induced ethylene production in Rumex palustris: regulation and ecophysiological significance. Plant J. 33, 341352. doi: 10.1046/j.1365-313x.2003.01632.x

Vriezen, W. H., Hulzink, R., Mariani, C., and Voesenek, L. A. (1999). 1aminocyclopropane-1-carboxylate oxidase activity limits ethylene biosynthesis in Rumex palustris during submergence. Plant Physiol. 121, 189-196. doi: 10.1104/pp.121.1.189

Wang, K. L.-C., Yoshida, H., Lurin, C., and Ecker, J. R. (2004). Regulation of ethylene gas biosynthesis by the Arabidopsis ETO1 protein. Nature 428, 945950. doi: $10.1038 /$ nature 02516

Xie, Z., Lee, E., Lucas, J. R., Morohashi, K., Li, D., Murray, J. A. H., et al. (2010). Regulation of cell proliferation in the stomatal lineage by the Arabidopsis MYB FOUR LIPS via direct targeting of core cell cycle genes. Plant Cell 22, 23062321. doi: 10.1105/tpc.110.074609

Xu, S. L., Rahman, A., Baskin, T. I., and Kieber, J. J. (2008). Two leucine-rich repeat receptor kinases mediate signaling, linking cell wall biosynthesis and ACC synthase in Arabidopsis. Plant Cell 20, 3065-3079. doi: 10.1105/ tpc.108.063354

Yamagami, T., Tsuchisaka, A., Yamada, K., Haddon, W. F., Harden, L. A., and Theologis, A. (2003). Biochemical diversity among the 1-aminocyclopropane-1-carboxylate synthase isozymes encoded by the Arabidopsis gene Family. J. Biol. Chem. 278, 49102-49112. doi: 10.1074/ jbc.M308297200

Yang, K., Wang, H., Xue, S., Qu, X., Zou, J., and Le, J. (2014). Requirement for Atype cyclin-dependent kinase and cyclins for the terminal division in the stomatal lineage of Arabidopsis. J. Exp. Bot. 65, 2449-2461. doi: 10.1093/jxb/ eru139

Yin, J., Zhang, X., Zhang, G., Wen, Y., Liang, G., and Chen, X. (2018). Aminocyclopropane-1-carboxylic acid is a key regulator of guard mother cell terminal division in Arabidopsis thaliana. J. Exp. Bot. 70, 897-908. doi: 10.1093/jxb/ery413 
Yoon, G. M., and Kieber, J. J. (2013). 14-3-3 regulates 1-aminocyclopropane-1carboxylate synthase protein turnover in Arabidopsis. Plant Cell 25, 10161028. doi: $10.1105 /$ tpc.113.110106

Zemlyanskaya, E. V., Omelyanchuk, N. A., Ubogoeva, E. V., and Mironova, V. V. (2018). Deciphering auxin-ethylene crosstalk at a systems level. Int. J. Mol. Sci. 19, 4060-4015. doi: 10.3390/ijms19124060

Zhang, Z., Ren, J.-S., Clifton, I. J., and Schofield, C. J. (2004). Crystal structure and mechanistic implications of 1-aminocyclopropane-1-carboxylic acid oxidasethe ethylene-forming enzyme. Chem. Biol. 11, 1383-1394. doi: 10.1016/ j.chembiol.2004.08.012
Conflict of Interest: The authors declare that the research was conducted in the absence of any commercial or financial relationships that could be construed as a potential conflict of interest.

Copyright (C) 2019 Polko and Kieber. This is an open-access article distributed under the terms of the Creative Commons Attribution License (CC BY). The use, distribution or reproduction in other forums is permitted, provided the original author(s) and the copyright owner(s) are credited and that the original publication in this journal is cited, in accordance with accepted academic practice. No use, distribution or reproduction is permitted which does not comply with these terms. 